This is a postprint version of the following published document:

Martínez-Cortés, T., Fernández-Torres, M. A., Jiménez-Moreno, A., González-Díaz, I., Díaz-de-María, F., Guzmán-de-Villoria, J. A. \& Fernández, P. (2014). A Bayesian model for brain tumor classification using clinical-based features. In 2014 IEEE International Conference on Image Processing (ICIP). (pp. 2779 - 2783). IEEE

DOI: http://dx.doi.org/10.1109/ICIP.2014.7025562

(C) 2014 IEEE. Personal use of this material is permitted. Permission from IEEE must be obtained for all other uses, in any current or future media, including reprinting/republishing this material for advertising or promotional purposes, creating new collective works, for resale or redistribution to servers or lists, or reuse of any copyrighted component of this work in other works. 


\title{
A BAYESIAN MODEL FOR BRAIN TUMOR CLASSIFICATION USING CLINICAL-BASED FEATURES
}

\author{
Tomás Martínez-Cortés ${ }^{1}$, Miguel Ángel Fernández-Torres ${ }^{1}$, Amaya Jiménez-Moreno ${ }^{1}$, \\ Iván González-Díaz ${ }^{1}$, Fernando Díaz-de-María ${ }^{1}$, Juan Adán Guzmán-De-Villoria ${ }^{2}$, Pilar Fernández ${ }^{2}$ \\ ${ }^{1}$ Department of Signal Theory and Communications, Universidad Carlos III, Leganés (Madrid), Spain \\ ${ }^{2}$ Hospital Gregorio Marañón, Madrid, Spain
}

\begin{abstract}
This paper tackles the problem of automatic brain tumor classification from Magnetic Resonance Imaging (MRI) where, traditionally, general-purpose texture and shape features extracted from the Region of Interest (tumor) have become the usual parameterization of the problem. Two main contributions are made in this context. First, a novel set of clinical-based features that intend to model intuitions and expert knowledge of physicians is suggested. Second, a system is proposed that is able to fuse multiple individual scores (based on a particular MRI sequence and a pathological indicator present in that sequence) by using a Bayesian model that produces a global system decision. This approximation provides a quite flexible solution able to handle missing data, which becomes a very likely case in a realistic scenario where the number clinical tests varies from one patient to another. Furthermore, the Bayesian model provides extra information concerning the uncertainty of the final decision.

Our experimental results prove that the use of clinical-based feature leads to a significant increment of performance in terms of Area Under the Curve (AUC) when compared to a state-of-the art reference. Furthermore, the proposed Bayesian fusion model clearly outperforms other fusion schemes, especially when few diagnostic tests are available.
\end{abstract}

Index Terms - Brain Tumor, MRI, clinical-based features, Bayesian fusion.

\section{INTRODUCTION}

Magnetic Resonance Imaging (MRI) is the diagnostic tool that currently offers the most sensitive non-invasive way for detection and diagnosis of brain neoplasms. According to the World Health Organization (WHO), brain tumors characterization is based on both their histological grade of malignancy (I-IV), and their type (primary or secondary) [1]. While the classification of the tumor grade is often simple for an experienced radiologist, the differentiation between more aggressive primary tumors (grade IV) and metastases could be more challenging [2]. In addition, this accurate diagnosis is essential because the management and prognosis of these type of tumors are very different [3].

Computer-Aided Diagnosis systems (CAD) have been developed that help physicians to make more accurate decisions. Typically, these systems use a Region Of Interest (ROI) segmentation method, followed by a feature extraction/selection procedure that feeds machine learning algorithms carrying out the lesion classification

Concerning the description of the ROI, most of these systems make use of traditional general-purpose texture-based image features: (Laplacian of Gaussian Filters [4], Gabor Filters [5], GLCM statistics [6], LBP/VAR histograms [7], etc.). These features model the texture of the ROI by means of filter banks at several scales and orientations, and have been successfully used in many computer vision tasks [8] [9] [10]. It has not been until the last years, when some recent approaches have introduced some specific clinical-based descriptors [11][12]. These type of descriptors are directly derived from the intuitions of the radiologists, and therefore model their expert knowledge about the problem. However, they were still very few in comparison to the general-purpose features, and sometimes they lacked of strong connections with the pathological information that radiology experts use in their diagnostics.

Another common limitation of current CAD systems for brain tumor type characterization is their lack of flexibility since they require exactly the same fixed set of clinical tests in all the cases (in most approaches, tumor area in T1 and T2). Therefore, in case an additional information is available, it is filtered out by the system. In contrast, if any of the images belonging to the fixed input set is not available (it has been not possible for the radiologists to make the associated clinical test), traditional CAD systems cannot make decisions about the case. However, in practice, many other MRI sequences (Fluid Attenuation Inversion Recovery or FLAIR, T1 after intravenous contrast agent administration) and some potential pathological indicators (enhancement, necrosis, edema, neovascularization, bleeding, etc.) are studied by physicians, and are frequently used to make their diagnosis.

To overcome the aforementioned limitations, in this paper we present a system for brain tumor classification with two important contributions: 1) we propose a rich set of clinical-based features that model properties of various pathological indicators used by physicians in their diagnosis. As we will show in the experimental section, this new set of features improves the traditional system performance working with general-purpose features. And 2) we propose a novel Bayesian fusion scheme that integrates the outputs of independent classifiers working on particular MRI sequences and pathological indicators. Bayesian fusion successfully handles the absence of information (missing clinical tests), and becomes a natural framework to model the uncertainty around the predictions what, as we will show in the paper, provides valuable information to physicians for both decision making and staff training purposes.

The remainder of this paper is organized as follows: Section 2 introduces our model for brain tumor classification. Section 3 focuses on the design of a novel set of clinical-based features and justifies each descriptor from the experts' knowledge. Our Bayesian fusion scheme is later discussed in Section 4. Section 5 assesses the performance of both the parameterization and global system, whereas Section 5 draws our conclusions and states further work.

\section{MODEL OVERVIEW}

Our work considers 4 types of MRI sequences that are usually included in the basic protocol used in the assessment of cerebral tu- 


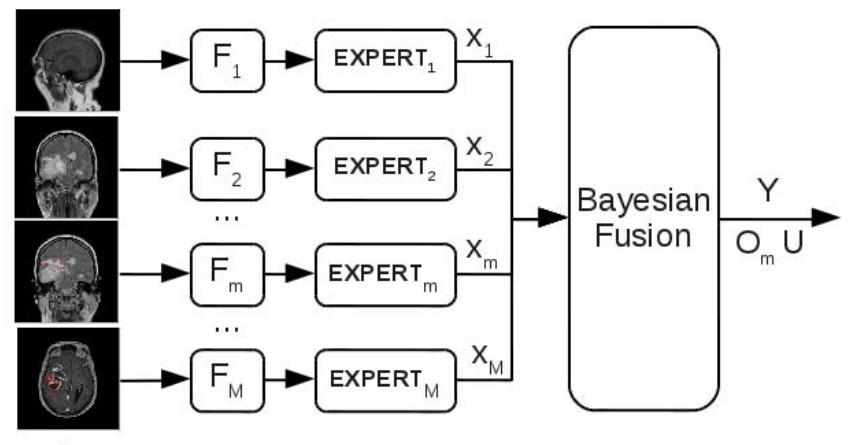

Pair $\mathrm{m}$

\{Seq, Ind $\}$

Fig. 1. Block diagram of the proposed model for MRI-based brain tumor classification.

mors: T1, T2, FLAIR and T1 3D after administration of intravenous gadolinium-based contrast, as well as 6 pathological image variables or indicators selected by expert radiologists that reflect histological features of these tumors: tumor area, enhancement, necrosis, edema, neovascularization and bleeding. The proposed model for MRIbased brain tumor classification is illustrated in Fig. 1. For each clinical case, the physicians generate one or more pairs (sequence, indicator) by manually labeling the ROI of each indicator (when it is present according to the radiologist criterion) in that sequence where the indicator is more noticeable or is particularly well-defined. Since our system is able to handle missing data, it is not required that all pairs (sequence, indicator) are available to make a decision about a clinical case. In fact, in practice, just a few subset of the potential pairs is available for each clinical case (in average, 5.1 in our dataset), and some of them are not even present in the whole dataset.

In the next step of the processing pipeline, the appearance of the ROI in each labeled pair (sequence, indicator) $m$ is described using a set of features $F_{m}$ (sec. 3). A particular (optimized) parameterization $F_{m}$ is obtained for each pair following a feature selection process (sec. 5.1). Then, the resulting feature vector $F_{m}$ will feed a classifier, which we call an expert, implemented by a SVM with a nonlinear RBF kernel. Each expert generates a real-valued output $X_{m}$, that represents the expert opinion, and corresponds with the soft decision function of the SVM: $X_{m} \in(-\infty, \infty)$.

Finally, the whole set of available expert decisions $X_{m}$ is then processed by a Bayesian Fusion model (sec. 4) that provides three measures of interest for the clinical case: a) the system decision $Y$; b) a set of individual expert opinions $O_{m}$; and c) the uncertainty $U$ of the decision.

\section{CLINICAL-BASED PARAMETERIZATION}

In this section, we introduce the features that describe the appearance of the ROI in each pair (sequence,indicator). In general, we consider two kinds of features: a) a set of classical general-purpose filter banks, e.g. Laplacian of Gaussian Filters [4], Gabor Filters [5], GLCM statistics( homogeneity, contrast, energy and correlation ) [6], LBP/VAR histograms [7], fractal dimension and lacunarity [13], entropy, range and standard deviation filters; and b) a set of clinicalbased features, containing specifically tailored features that model perceptions and intuitions of radiologists and other clinical experts.

Since they are a central point in the scope of this paper, we next provide a description of the clinical-based features, focusing on how they are derived from the experts' intuitions. Hence, given the ROI of an indicator in a sequence, we compute:
- Size relations: computed as areas, perimeters and their relations to those of other indicators. Size of Primary tumors tends to be bigger when compared to other associated pathological evidences ( edema, enhancement, etc ).

- Scattering and complexity: measures the number of nonconnected regions that form a particular indicator along with their shape regularity. Primary tumors are characterized by irregular perimeters with scattered enhancement; whereas metastases are generally more regular with a single region of enhancement. This feature are related to the more infiltrative nature of gliomas [14]. Complexity measure includes regularity and three types of asymmetry (rotational, mirror and circular) ) reflecting the histological inhomogeneity of primary brain tumors [15].

- Compactness and annularity: these are features designed to describe the whole enhancement and necrosis area. Metastasis tends to have circular necrosis areas surrounded by annular enhancement, while primaries are more decentralized and snaky.

- Edge intensity: this feature describes the transition between the boundary of the lesion and the healthy tissue. Primary tumors are mainly composed by neurons which are visually similar to the rest of the gray matter, but metastases are formed by cells from other organs, so the intensity gradient along the border tends to be larger.

\section{BAYESIAN FUSION OF EXPERT OPINIONS}

\subsection{Independent likelihood pool for fusion of expert opinions}

Given a particular clinical case, the input to the fusion stage is a feature vector $X=\left\{x_{1}, x_{2}, \ldots, x_{M}\right\}$ containing the soft outputs of the $M$ experts available for the case. The aim of this stage is to generate a final output label $Y$, a measure of the uncertainty $U$ of this decision, and a measure of the experts' individual opinions $O_{m}$.

Bayesian models are a suitable paradigm to carry out the fusion process of the outputs of several experts. Assuming that, conditioned to the class $j$ ( $j=0,1$ for primary and metastasis, respectively), the likelihood of the data provided by $m$-expert $p\left(x_{m} \mid y_{j}\right)$ is independent of that of any other different expert $n(\forall n \neq m)$, we can easily factorize the joint likelihood of the experts' opinion $p\left(X \mid y_{j}\right)$. Hence, using Bayes' theorem, we can model the posterior probability of a class $j$ :

$$
p\left(y_{j} \mid x_{1} \ldots x_{M}\right) \propto p\left(y_{j}\right) \prod_{m=1}^{M} p\left(x_{m} \mid y_{j}\right),
$$

that depends on the prior probability of the class $p\left(y_{j}\right)$, and the likelihood of the inputs $p\left(x_{m} \mid y_{j}\right)$.

This model is known as Independent Likelihood Pool [16] and, due to the factorization of the likelihood, allows us to implicitly handle missing clinical tests.

As can be observed in (1), we need to define the distribution that models the likelihoods of the expert outputs $p\left(x_{m} \mid y_{j}\right)$. We used Gaussian distributions to model the expert outputs: $p\left(x_{m} \mid y_{j}\right)=$ $N\left(\mu_{j m}, \sigma_{j m}\right)$, defined by their mean $\mu_{j m}$ and standard deviation $\sigma_{j m}$. Modeling the expert outputs as Gaussian distributions, rather than directly using their values, allows the model, not only to handle missing data, but also to measure the uncertainty over the outputs of the SVMs.

Up to now, the proposed Bayesian fusion scheme has modeled just one source of uncertainty (that one directly coming from the quality of the individual SVMs). However, we also aim to incorporate an additional source of uncertainty: that one coming from the lack of enough training samples. To that end, we propose to introduce a priori distributions $\left(\mu_{0}, \sigma_{0}\right)$ over the parameters of the Gaussian 

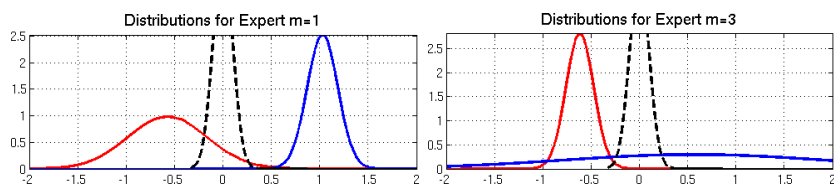

Fig. 2. Examples of the distributions for 2 experts: the prior distribution is shown in black dashed line, that of the metastasis class in blue line, and that of the primary tumor class in red line.

likelihoods. For simplicity, conjugate prior distributions are used to analytically derive closed expressions for the posteriors. Specifically, the conjugate prior of the precision $\left(\lambda_{j m}=\sigma_{j m}^{-2}\right)$ is a Gamma distribution, whereas the conjugate prior of the mean $\mu_{j m}$ is a Gaussian distribution conditioned on $\lambda_{j m}[17]$.

For the sake of brevity, we omit the algebra related to conjugate priors (the reader is referred to [18] and [19] for details). The resulting update equations to estimate the Gaussian parameters are:

$$
\begin{aligned}
\hat{\mu}_{j m} & =\frac{\sum_{i=1}^{n_{j m}} x_{j m}^{(i)}+\beta \mu_{0}}{n_{j m}+\beta} \\
\hat{\sigma}_{j m}^{2} & =\frac{\sum_{i=1}^{n_{j m}}\left(x_{j m}^{(i)}-\hat{\mu}_{j m}\right)^{2}+\beta\left(\left(\hat{\mu}_{j m}-\mu_{0}\right)^{2}+\sigma_{0}^{2}\right)}{n_{j m}+\beta}
\end{aligned}
$$

where $n_{j m}$ stands for the number of available data for the $m$-expert and the class $j ; \mu_{0}$ and $\sigma_{0}^{2}$ are the initial values for the Gaussian parameters (in our experiments, $\mu_{0}=0$ and $\sigma_{0}^{2}$ small $\left(\sigma_{0}^{2} \sim 0.1\right.$ ); and finally, the $\beta$ parameter is a scaling factor that balances the influence of the initial and the learned models (in our experiments, $\beta=1$ ). It should be noted that all the distributions, each one associated with an expert and a class, have the same initial values $\mu_{0}, \sigma_{0}^{2}$, and scaling factor $\beta$.

The underlying idea is that the accuracy of the Gaussian models of the expert outputs increases with each new training data. In doing so, we are able to manage the shortage of training data. For example, if an expert provides us few training data $p\left(x_{m} \mid y_{j}\right)$ (because this expert works with a sequence and indicator rarely labeled by the physician), the Gaussian distributions associated with this expert will be very similar to those of the a priori distributions; and vice-versa, if an expert gives us many training data, the Gaussian distributions will highly depend on these data and the influence of the a priori distribution will be lower.

In Fig. 2 two examples of this learning process are shown, each one for a different expert. Specifically, the distributions of each expert output given each class (primary tumor and metastasis) are shown along with the a priori distributions. As can be observed, when enough data is available the distributions clearly move apart from the a priori distributions. Otherwise, they would stay close to the a priori distributions expressing a high level of uncertainty. Moreover, the actual overlap between the distributions for primary tumor or metastasis for each expert clearly indicates its discrimination ability. In this particular case, expert 1 will likely discriminate much better than expert 3 .

In summary, the proposed method is able to model both the variability of the expert opinion and the shortage of training data. Moreover, if some sequence or indicator is not available, this absence can be managed by simply not including the corresponding term in (1).
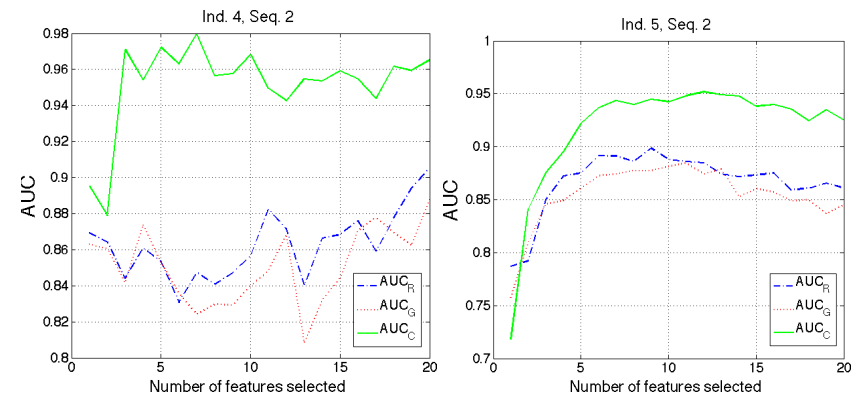

Fig. 3. Evolution of the AUC as a function of the number of features selected for pairs $\{4,2\}$ and $\{5,2\}$. $A U C_{C}$ denotes the proposed feature set.

\subsection{Further information provided by the Bayesian fusion model}

Besides the final selected class, the previously described model allows us to obtain some further information of great interest, such as the uncertainty of its decision or the individual opinion of every expert. These kind of additional information may be useful to assist the physician who has to make the decision and even to contribute to the education process of inexperienced physicians. In particular, we propose to compute the following additional measures for each clinical case.

Final system decision $Y$ : it is calculated following (1); thus, if the probability of primary tumor is higher than the probability of metastasis $p\left(y_{0} \mid x_{1} \ldots x_{M}\right)>p\left(y_{1} \mid x_{1} \ldots x_{M}\right)$, the selected class will be primary tumor $Y=0$, and vice-versa.

Individual expert opinions $O_{m}$ : computed as:

$$
O_{m}=p\left(y_{j} \mid x_{m}\right) \propto p\left(y_{j}\right) p\left(x_{m} \mid y_{j}\right)
$$

being $j$ the final selected class. In particular $O_{m}$ tells how good the decision would be if only the $m$-expert were used. Therefore, these expert opinions lets us to know the sequences and indicators that become essential to the final decision.

Uncertainty $U$ of the final decision: defined as the mean deviation between the probability of every $m$-expert $p\left(y_{j} \mid x_{m}\right)$ and the final output of the fusion stage $p\left(y_{j} \mid x_{1} \ldots x_{M}\right)$, where $j$ is the selected class:

$$
U=\sqrt{\frac{1}{M} \sum_{m=1}^{M}\left(p\left(y_{j} \mid x_{1} \ldots x_{M}\right)-p\left(y_{j} \mid x_{m}\right)\right)^{2}}
$$

This uncertainty measure provides some insight into the reliability of the system decision.

\section{EXPERIMENTAL RESULTS}

\subsection{Experimental Setup}

Our dataset contains 97 clinical cases, 85 of them corresponding to the most frequent type of primary tumors (gliomas) and 12 to metastases. As previously mentioned, the average number of experts per patient is 5.1. The number of training data available for each expert model varies according to the sequences and indicators that the physicians decided to label for each clinical case. Furthermore, some experts have been discarded since there are not enough metastases to train and evaluate the corresponding models. In particular, in our experiments we only consider those experts for which we have at least 10 primary tumors and 5 metastases, what leads to a set of 8 experts in total. 


\begin{tabular}{|l|l|c|c|c|c|}
\hline Ind. & Seq. & $\boldsymbol{\Delta} \boldsymbol{A} \boldsymbol{U} \boldsymbol{C}_{\boldsymbol{R}}$ & $\boldsymbol{\Delta A U \boldsymbol { C } _ { \boldsymbol { G } }}$ & $\boldsymbol{A U} \boldsymbol{C}_{\boldsymbol{C}}$ & \# clinic/\# features \\
\hline 2 & 2 & 3.49 & 4.00 & 99.19 & $13 / 19$ \\
\hline 2 & 3 & 1.86 & 1.68 & 99.22 & $7 / 11$ \\
\hline 2 & 5 & 6.41 & -3.59 & 88.20 & $5 / 6$ \\
\hline 3 & 5 & 4.44 & 1.09 & 87.19 & $6 / 15$ \\
\hline 4 & 2 & 8.93 & 10.10 & 97.97 & $4 / 5$ \\
\hline 4 & 5 & 3.26 & 2.20 & 93.89 & $4 / 10$ \\
\hline 5 & 2 & 5.05 & 6.30 & 95.19 & $3 / 14$ \\
\hline 5 & 3 & -0.36 & 0.71 & 86.44 & $1 / 9$ \\
\hline
\end{tabular}

Table 1. Results regarding the compared feature sets for the 8 pairs \{sequence, indicator considered. The AUC achieved by the proposed system, $A U C_{C}$, and the increments with respect to the other two feature sets considered, $\triangle A U C_{R}$ and $\triangle A U C_{G}$, are shown.

We have conducted our experiments following a well-defined protocol. For each individual expert, we have designed the corresponding model using a 5-fold cross validation, which has been used to perform both the feature selection process and the selection of the parameters of the SVM ( $C$ and $\gamma$, the width of our RBF kernel).

Then, due to the limited size of the individual expert datasets, we have evaluated our classifiers using the Leave-One-Out (LOO) approach. The advantage of LOO is twofold: 1) it obtains more stable results since it maximizes the number of training samples for every test sample; and 2) it obtains results for every sample in the dataset, what allows us to train the fusion model over the whole set of clinical cases. Finally, we have used the Area Under the Curve (AUC) to assess the performance of our approach.

\subsection{Experiments on feature sets}

In this section we have assessed how the inclusion of clinicalbased features actually improves the system performance. Hence, we have compared two feature sets: a General set $(\mathrm{G})$ that includes classical texture filter-banks (see Sec. 3), and a Complete set (C), that incorporates the proposed novel set of clinical-based features (Sec. 3 ). Moreover, to provide comparisons with an state-of-the-art method, we have considered in our experiments a third feature set proposed in [20] and [21] (R), which comprises many general-purpose filter-banks and a reduced set of clinical-based features.

In order to analyze the evolution of the AUC as a function of the number of features selected, we have repeated the whole process 50 times, varying the dimension of the feature vector from 1 to 20 , and compared the results achieved by the three considered feature sets. Table 1 summarizes these results for each expert, i.e., for each pair (sequence, indicator). To evaluate the improvement reached by the proposed set $(\mathrm{C})$, the mean increment of AUC with respect to the state-of-the art reference $\left(\triangle A U C_{R}\right)$ and to the general purpose feature-based systems $\left(\triangle A U C_{G}\right)$ has been measured (third and fourth column). The fifth column includes the best AUC obtained by the optimal set of features. And finally, the last column shows the number of slected clinical-based features with respect to the total number of features in the optimal feature set.

Figure 3 illustrates, as an example, the evolution of the AUC as a function of the the number of selected features for two particular pairs (sequence, indicator), $\{2,2\}$ and $\{5,2\}$.

As can be seen in Figure 3 and Table 1, the use of clinical-based features produces a notable increment of the AUC with respect to both the general-purpose and the reference sets for the same number of features selected. Furthermore, the proportion of clinical-based features over the total number of features is very significant in almost every case.

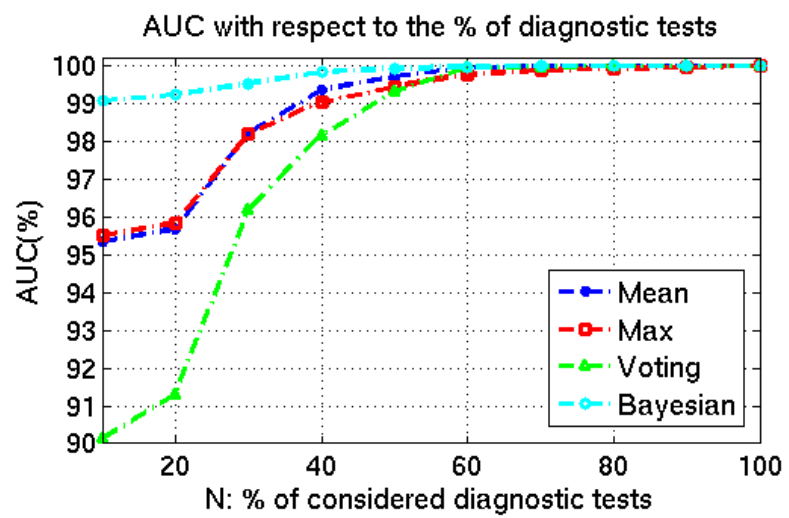

Fig. 4. Performance of the proposed model for Bayesian fusion in comparison with max, mean, and max-voting fusion schemes.

\subsection{Experiments on the global system}

Once our clinical-based parameterization has been assessed, we aim to demonstrate how the proposed fusion scheme handles the problem of missing data (absent clinical tests) and models the uncertainty due to the scarcity of training data and the confidence on each expert.

To this purpose, we have evaluated the performance of the global system at classifying clinical cases for a different number of available diagnostic tests (experts). In particular, given the whole dataset, we randomly pick $N \%$ of the diagnostic tests for each case (ensuring at least we have 1 diagnostic test per case) and run our system. In Fig. 4 we show the results of this experiment, comparing the performance of our Bayesian fusion scheme to other well known fusion schemes that can also handle missing data, namely: mean fusion, max fusion and max-voting fusion.

As can be easily noticed, the proposed Bayesian fusion model consistently outperforms the rest of the approaches. It is worth noting how the performance of our fusion scheme is specially good when the number of diagnostic tests is very low. From our point of view, the rationale behind this fact is that for these values of $N$, the number of training samples for each expert is very low, what produces an important increment of the uncertainty of the individual SVM-based classifiers. Since our approach is the only one modeling this uncertainty using Gaussian distributions and conjugate priors, the confidence on the individual expert decisions is taken into account.

\section{DISCUSSION}

In this paper, we have proposed a complete system for brain tumor classification from MRI-based images. First, we have introduced some clinical-based features that intend to model radiologists' intuitions and, as shown in the experiments, notably improve the results, either increasing the classification performance or reducing the total number of features required for classification.

Second, we have proposed a Bayesian fusion model that successfully handles the problem of missing data, takes into account the uncertainty of each expert, and provide useful information to the radiologists. That is especially important because we consider a realistic scenario where although an extended set of potential diagnostic tests could be performed, just a small set of them is actually available for each clinical case.

Further work focuses on the development of a larger dataset and the development of more elaborated Bayesian fusion methods. 


\section{REFERENCES}

[1] D.N. Louis, B.W. Scheithauer, H. Budka, and J.J. Kepes, World Health Organization Classification of Tumors. Pathology and Genetics. Tumors of the Nervous System, IARC Press, Lyon, France, 2004.

[2] Stella Blasel, Alina Jurcoane, Kea Franz, Gerald Morawe, Stefanie Pellikan, and Elke Hattingen, "Elevated peritumoural rcbv values as a mean to differentiate metastases from highgrade gliomas.," Acta Neurochir (Wien), vol. 152, no. 11, pp. 1893-9, 2010.

[3] S Wang, S Kim, S Chawla, R L Wolf, D E Knipp, A Vossough, D M ;Rourke, K D Judy, H Poptani, and E R Melhem, "Differentiation between glioblastomas, solitary brain metastases, and primary cerebral lymphomas using diffusion tensor and dynamic susceptibility contrast-enhanced $\mathrm{mr}$ imaging.," AJNR Am J Neuroradiol, 2011.

[4] T. Lindeberg, "Scale-space theory: A basic tool for analysing structures at different scales," J. of Applied Statistics, vol. 21(2), pp. 224-270, 1994.

[5] Mahamadou Idrissa and Marc Acheroy, "Texture classification using gabor filters," Pattern Recognition Letters, vol. 23, no. 9, pp. 1095 - 1102, 2002.

[6] R.M. Haralick, K. Shanmugam, and Its'Hak Dinstein, “Textural features for image classification," Systems, Man and Cybernetics, IEEE Transactions on, vol. SMC-3, no. 6, pp. 610-621, 1973.

[7] Timo Ojala, Matti Pietikäinen, and David Harwood, "A comparative study of texture measures with classification based on feature distributions," Pattern Recognition, vol. 29, no. 1, pp. 51-59, 1996.

[8] D.M. Joshi, N. K. Rana, and V. M. Misra, "Classification of brain cancer using artificial neural network," in Electronic Computer Technology (ICECT), 2010 International Conference on, May 2010, pp. 112-116.

[9] V. Kumar, J. Sachdeva, I. Gupta, N. Khandelwal, and C.K. Ahuja, "Classification of brain tumors using pca-ann," in Information and Communication Technologies (WICT), 2011 World Congress on, Dec 2011, pp. 1079-1083.

[10] D. Sridhar and I.V. Murali Krishna, "Brain tumor classification using discrete cosine transform and probabilistic neural network," in Signal Processing Image Processing Pattern Recognition (ICSIPR), 2013 International Conference on, Feb 2013, pp. 92-96.

[11] E.I. Zacharaki, Sumei Wang, S. Chawla, Dong Soo Yoo, R. Wolf, E.R. Melhem, and C. Davatzikos, "Mri-based classification of brain tumor type and grade using svm-rfe," in Biomedical Imaging: From Nano to Macro, 2009. ISBI '09. IEEE International Symposium on, June 2009, pp. 1035-1038.

[12] Megha.P. Arakeri and G.RamMohana Reddy, "Computeraided diagnosis system for tissue characterization of brain tumor on magnetic resonance images," Signal, Image and Video Processing, pp. 1-17, 2013.

[13] O.S. Al-Kadi and D. Watson, "Texture analysis of aggressive and nonaggressive lung tumor ce ct images," Biomedical Engineering, IEEE Transactions on, vol. 55, no. 7, pp. 1822-1830, 2008 .
[14] Stanley Lu, Daniel Ahn, Glyn Johnson, Meng Law, David Zagzag, and Robert I. Grossman, "Diffusion-tensor MR imaging of intracranial neoplasia and associated peritumoral edema: introduction of the tumor infiltration index.," Radiology, vol. 232, no. 1, pp. 221-228, July 2004.

[15] Carmine Franco Muccio, Annachiara Tarantino, Gennaro Esposito, and Alfonso Cerase, "Differential diagnosis by unenhanced flair 2 -weighted magnetic resonance images between solitary high grade gliomas and cerebral metastases appearing as contrast-enhancing cortico-subcortical lesions.," J Neurooncol, vol. 103, no. 3, pp. 713-7, 2011.

[16] James O. Berger, Statistical decision theory and Bayesian analysis, Springer series in statistics. Springer, New York, NY [u.a.], 2. ed edition, 1985.

[17] P.M. Lee, Bayesian Statistics: An Introduction, Wiley, 2012.

[18] Kevin P. Murphy, Conjugate bayesian analysis of the gaussian distribution, Technical report, UBC, 2007.

[19] J. Goldberger and H. Greenspan, "Context-based segmentation of image sequences," Pattern Analysis and Machine Intelligence, IEEE Transactions on, vol. 28, no. 3, pp. 463-468, March 2006.

[20] J. Sachdeva, V. Kumar, I. Gupta, N. Khandelwal, and C.K. Ahuja, "Multiclass brain tumor classification using ga-svm," in Developments in E-systems Engineering (DeSE), 2011, Dec 2011, pp. 182-187.

[21] Jainy Sachdeva, Vinod Kumar, Indra Gupta, Niranjan Khandelwal, and Chirag Kamal K. Ahuja, "Segmentation, Feature Extraction, and Multiclass Brain Tumor Classification.," Journal of digital imaging, May 2013. 